\title{
A combined change detection procedure to study desertification using opensource tools
}

\author{
Anna Zanchetta* ${ }^{*}$ and Gabriele Bitelli
}

\begin{abstract}
Background and Methods: The paper presents a combination of two unsupervised techniques for change detection studies in arid and semi-arid areas. Among Remote Sensing change detection techniques, unsupervised approaches have the advantage of promptly producing a map of the change between two dates, but often the interpretation of the results is not straightforward, and requires further processing of the image. The aim of the research is to propose a new time effective and semi-automated reproducible technique in order to reduce the weakness of the unsupervised approach in change detection. Two techniques, Change Vector Analysis (CVA) and Maximum Autocorrelation Factor transform of Multivariate Alteration Detector components (MAD/MAF) are chosen to serve the purpose.

Results and Conclusions: The results of the research, applied to two case studies in the Middle East region, indicate that the chosen techniques complement each other, since MAD/MAF gives a detailed spatial extent while CVA gives the semantic interpretation of the output. The research brings further understanding to the use of both unsupervised procedures and the methodology can be used as a fast semi-automatic preliminary step for more accurate change detection studies. A further output is a new add-on implementing CVA for the GFOSS (Geospatial Free and Open Source Software) project Grass GIS.
\end{abstract}

Keywords: GFOSS, Grass GIS, Orfeo Toolbox, Change Vector Analysis, Tasselled Cap Transform, Multivariate Alteration Detector, Maximum Autocorrelation Factor

\section{Background}

In Remote Sensing (RS) of the environment, change detection covers the capability to identify the differences occurred on the Earth surface in time. This task can be performed through various techniques, and choosing between them depends also on the characteristics of the surface that need to be detected. Often change detection analysis is referred to as Land use/Land Cover Change (LULCC) analysis, referring to the characterization of the terrestrial surface in terms of natural and man built types of cover. A basic condition for change detection studies is the preliminary co-registration of the images and the correction for external factors of change on the surface reflectance, such us atmospheric conditions and differences in Sun angle, plus the intrinsic characteristics of the single sensors. The selection of appropriate images, in

\footnotetext{
* Correspondence: anna.zanchetta@unibo.it

DICAM Dipartimento di Ingegneria Civile, Chimica, Ambientale e dei Materiali, Università di Bologna, Viale Risorgimento 2, Bologna, Italy
}

terms of time of the year and weather conditions, allows to reduce the impact of these factors [1].

LULCC analysis requires in general a first identification of the surface covers, then a mechanism that compares the images along a temporal interval. The identification of the different types of cover is called image classification and can be carried out through several techniques and procedures [1-3]. Most techniques process the single pixel and are so called pixel-based techniques. They differentiate from the relatively recent object-based analysis, that groups the pixels not only depending on their spectral behavior but also on geometrical characteristics, such as shape, color, size and similarity with neighboring pixels [4].

Considering pixel-based procedures, the categorization of the different types of classification techniques varies widely from one author to another, but mainly two different approaches can be recognized: supervised and unsupervised classification. In the first approach, supervised classification, the single images to be compared are classified separately, 
based on a preliminary knowledge of training areas for the different classes. The user identifies on the image some polygons of interest that correspond to specific land cover types. These training areas are then assigned to a software, that recognizes the spectral characteristics of each class and performs, through a selected algorithm, the classification of the rest of the image. The algorithms include maximum likelihood and minimum distance classification, among others.

Unsupervised procedures on the other hand are used in absence of samples or previous information on the ground. In this case the software makes use of the spectral characteristics of the pixels in order to group them in different classes. The techniques for unsupervised classification include clustering techniques like the ones used in unsupervised classification (maximum likelihood, minimum distance, k-means, ISODATA etc.) and also techniques that make use of simple algebraic procedures or more complex statistical and mathematical analysis procedures as a preliminary step for the classification. The algebraic procedures include differencing, ratioing, indexes (like Normalized Difference Vegetation Index - NDVI), while the mathematical analysis techniques include data transformations (like Principal Component Analysis - PCA, Tasselled Cap Transform - TCT, Multivariate Alteration Detector - MAD), Artificial Neural Network (ANN) analysis, fuzzy logic, Bayesian network and others. Some unsupervised procedures promptly give as output a map of the change that must then be interpreted assigning a threshold, in order to differentiate the significant change from the pixels which are unchanged. The threshold choice, through customary or statistical criteria, is the key for the change interpretation $[5,6]$.

The choice of the type of techniques to be used depends on the purpose of the research and on the availability of ground truth data, accessory material and knowledge of the studied area. Supervised classification is an ideal procedure, but often finding both spatial and semantic accuracy can be challenging and relies on the skills of the classifier. Moreover the availability of knowledge on the field can not always be complied and an accurate classification can be time consuming. On the contrary, unsupervised approaches can be almost automatic and more time-efficient, but require the researcher to intervene in a second phase to interpret the results. Therefore, according to the aim of the researcher, the change detection analysis can be performed using a mixture of more techniques, the so-called hybrid approach, that combines two or more techniques in order to improve the results $[6,7]$.

For this research, which was missing ground truth data, two semi-automated unsupervised techniques have been chosen: the Change Vector Analysis (CVA) applied to the TCT features, and the Maximum Autocorrelation Factor (MAF) transformation of the MAD.
CVA, developed in the 80s [8], gives as an output the direction of the change between two dates for each pixel of an image, having the advantage of not needing an image classification to be performed in advance. The CVA needs in input two (or more) spectral characteristics (bands or spectral features) of the surface, that have a physical meaning and whose combination in the bands space can give a physical interpretation of the change. For this aim TCT features are ideal candidates and have been widely used in several RS studies [8-11]. In contrast to PCA, which must be calculated for each single image, TCT is meant to be image independent, meaning that it can be applied to any image, provided that the TCT coeffiecient have been previously calculated for its specific satellite sensor.

The second RS methodology used in the present study is the combined MAD/MAF analysis. MAD transformation was introduced by Nielsen et al. [12] to improve the simple image differencing technique, by considering the difference images with maximal variance, obtaining a new set of uncorrelated images. Although similar, MAD was intended to be an improvement over PCA transform [12]. PCA in fact is a statistical procedure used, not only in RS but in several fields of Science, to transform a set of correlated variables to a new set of uncorrelated variables (called components) that consider the principal directions in which the data are spread in the bands space. PCA is useful to reduce the size and redundancy in the original data, since most of the information is contained in the first components, while the latter ones contain mainly noise. To do so, PCA considers the maximum variance in the dataset, whereas MAD considers maximum autocorrelation, since it takes into account the maximum variance of the difference images. Doing such, MAD eliminates issues related to the possibility that a dominating element in the image affects the PCA components, with a disproportioned high variance compared to other elements of the image. Besides this, MAD has the huge advantage of being invariant for linear transformations of the data, making it insensitive to the application to raw DN or transformed images [12, 13].

Like PCA, though, MAD analysis is locally applied to the single pixel and therefore doesn't retain the spatial context of the adjacent pixels. With this consideration in mind, Nielsen introduced the application of the MAF transform to the MAD components, which is intended to produces variates ordered by spatial autocorrelation [14]. The MAD/MAF method thus provides a "statistically rigorous system to determine the spatially coherent patterns of major change in an image sequence" [12].

Several comparison studies have been carried out on the performance of these three techniques, CVA, PCA and MAD, also in desertification studies $[2,15,16]$. In particular Pannenbecker [15] concluded that CVA and 
MAD were the most effective techniques, in terms of desertification indicators and in terms of results. Considering also the added value, described above, of the MAF applications to the MAD components, in our research we chose to use CVA and MAD/MAF analysis, and further developed them in order to improve the analysis by combining the results of the two.

In desertification studies, change detection covers a prominent role, since it allows the identification of surface elements that changed over a large scale, both spatial and temporal, and constitutes an effective method for observing, monitoring and characterizing the dynamism of drylands [17]. One of the biggest challenge in this type of studies is the identification of common desertification indicators, since different ways of interpreting analogous factors can bring to different results $[17,18]$. Several indicators have been proposed in literature, considering in particular the soil reflectance contribution and the vegetative mass, whose deterioration can be a good indicator of desertification, being the major biological production of desert areas [19]. In particular different authors used indicators of: land degradation (soil erosion, soil salinization [20]), land use changes (expansion of agricultural or urban areas), bare soil expansion, drought and changing vegetation (perennial plant cover and biomass) $[15,17,18]$. The usage of the change detection techniques commonly used for vegetated areas, requires though some care in applications to desert areas: since vegetative cover is low, the reflectance of the exposed soil surface highly influences the pixels value $[17,21]$. On the other hand, drylands have advantageous weather characteristics for change detection studies, exhibiting dry and cloudless conditions which remain unchanged for a long part of the year. These are ideal for change detection studies, both on a short (seasonal) and long (years) scale, solving the issue connected with the atmospheric correction, a basic prerequisite for change detection studies [1].

The aim of the research is to reduce the weakness of unsupervised techniques, mainly absence of ground truth information, by the joint use of two of these techniques in a hybrid approach, thus introducing a new and reproducible change detection procedure. In specific, the effectiveness of a combination of CVA and MAD/MAF is investigated in change detection studies in arid and semi-arid areas, naturally prone to desertification processes.

The research was conducted using Geospatial Free and Open Source Software (GFOSS). The use of GFOSS allowed to implement unavailable techniques and to adapt existing ones to the needs of the research. Several OSGEO (Open Source GEOspatial Foundation) products, namely Grass GIS, QGIS and Orfeo Toolbox, were used through user's specified scripts in BASH and Python. For the aim of this research, a new addon for Grass GIS performing CVA was also implemented.

\section{Methods \\ Landsat data}

To carry out the research, two case studies have been chosen in arid and semi-arid areas following the Koppen climate map [22], where arid areas (BW) and semi-arid areas (BS) are defined based on a combination of mean annual precipitation and mean annual temperature.

The first case study is Al Azraq Oasis, in Jordan. The Oasis is located in the hot desert Badia region, $90 \mathrm{~km}$ East of the capital Amman and was subject in the early 90 s to a rapid drying out of the water springs due to overexploitation of the groundwater [23, 24] .

The second case study is the lake originated by the South Hasakah Dam, in North East Syria, built $25 \mathrm{~km}$ South of Al Hasakah in the late 90s on the Khabur River, tributary of the Euphrates River. This reservoir is located in a very fertile rural area (so called Syrian bread basket) which has historically been highly exploited for the surface and groundwater resources $[25,26]$.

The two sites, which locations are shown in Fig. 1, are characteristic of desert environment and present water bodies and vegetated areas, mainly agricultural. In the case of Al Azraq, the area surrounding the Oasis shows also natural vegetation growing around the water sources.

For Al Azraq Oasis the information on the ground were given by historical maps, aerial photographies and personal communications collected during a field visit as discussed previously by the authors [23]. Furthermore, this previous study included a change detection analysis using some of the same techniques (CVA, TCT), and so the confrontation with the new methodology was direct.

Several Landsat satellites images were chosen before and after the 90s to assess the expected change in the two areas: in the first case the drying out of the Oasis and in the second case the appearance of the Lake. In order to reduce dissimilarities in the atmospheric and weather conditions in situ, the criteria for the choice of the images were based on similar acquisition date and summer season. Therefore images in the end of August 1984 and August 2015 were selected for the change detection analysis. The chosen images are listed in Table 1, alongside the denomination that will be used to refer to them in the rest of the paper. Table 2 lists the characteristic of the two used Landsat satellites sensors, Landsat 5 Thematic Mapper (TM) and Landsat 8 Operational Land Imager (OLI). Only the spectral bands used in the research are shown.

\section{The Tasselled Cap Transform (TCT) and Change Vector Analysis (CVA)}

TCT was developed by Kauth and Thomas [27] for Landsat 5 MSS sensor DN data. It owes its name to the particular shape they recognized in the bands space for the development of the pixels' values, considering the 


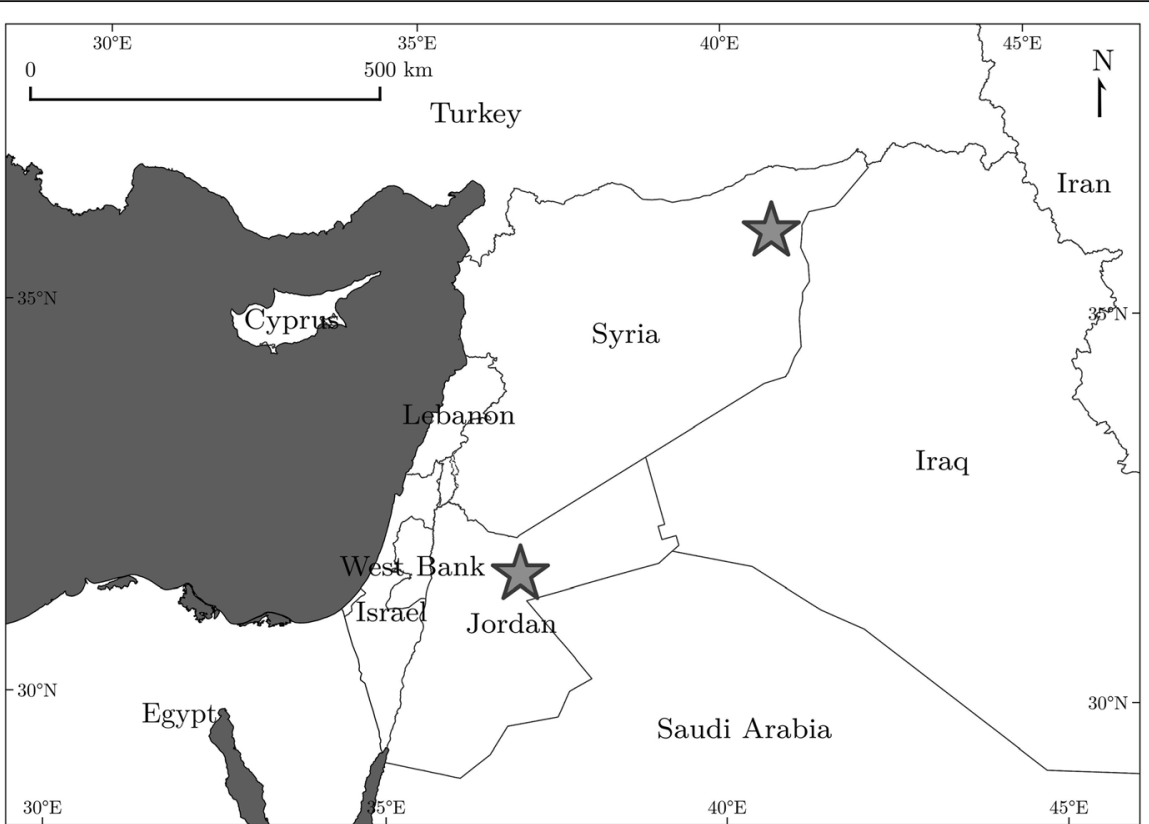

Fig. 1 Location of the two case studies (grey stars) in Syria and in Jordan in the Middle East region

life cycle of some crops in a rural area in Illinois, USA. The idea of TCT is to linearly transform through a set of coefficients the pixels' values in the original bands (normally highly correlated) to a new set of bands, that are orthonormal. The new bands are called features and have been originally developed in order to have a meaning in terms of different surface covers. For this reason the TCT is image independent and can be applied to different images, provided that the coefficients have been calculated for that type of satellite and data (raw digital number, Top Of Atmosphere Reflectance - TOAR, etc). TCT features' meaning is connected with characteristics such as: soil reflectance (first feature, called Brightness), vegetation (second feature, Greenness), wet areas (third feature, Wetness). Further features that can be retrieved with TCT, which are in fact as many as the initial bands, have been interpreted in different studies in several ways, so their meaning is not univocal $[28,29]$.

The first TCT features, Brightness and Greenness, are commonly used as input for the CVA [10, 11], as suggested by Malila itself [8], who developed it in 1980. Further studies used also the third feature, Wetness, for a

Table 1 Selected dates for Landsat 5 and Landsat 8 satellites for the multitemporal change detection

\begin{tabular}{llll}
\hline Date & Satellite & Path/row & Denomination \\
\hline 1984/08/30 & Landsat 5 & $173 / 38$ & Oasis 1984 \\
1984/09/01 & Landsat 5 & $171 / 35$ & Lake 1984 \\
2015/08/20 & Landsat 8 & $173 / 38$ & Oasis 2015 \\
2015/08/22 & Landsat 8 & $171 / 35$ & Lake 2015 \\
\hline
\end{tabular}

three dimensional CVA $[9,30]$, but for the aim of the research this option wasn't considered in the present study. In fact the canonical bi-dimensional CVA, applied to two spectral bands (or features) indicative of soil reflectance and vegetative conditions, as is the case of Brightness and Greenness, is effective in detecting changes towards drier or wetter conditions and can be used also in desert areas applications [17, 23, 31, 32].

CVA's concept is rather simple: it uses basic geometrical calculations to derive the change occurred to the value of a pixel in two (or more) spectral bands or spectral characteristics between two dates. In the bands space, plotting the values of the pixel in two different dates generates a vector, whose length and angle give the magnitude and direction of the change, respectively (see Fig. 2). The output of the CVA are two maps: the angle map bears a meaning in terms of change to the

Table 2 Technical data of selected spectral bands used in the research for the Landsat 5 satellite TM (Thematic Mapper) and Landsat 8 satellite OLI (Operational Land Imager) sensors, with resolution of $30 \mathrm{~m}$

\begin{tabular}{lllllll}
\hline \multirow{2}{*}{ Denomination } & \multicolumn{2}{l}{ Landsat 5 TM } & & \multicolumn{2}{l}{ Landsat 8 ETM+ } \\
\cline { 2 - 3 } & band\# & range $(\mu \mathrm{m})$ & & band\# & range $(\mu \mathrm{m})$ \\
\hline Blue & 1 & $0.45-0.51$ & & 2 & $0.45-0.52$ \\
Green & 2 & $0.52-0.60$ & 3 & $0.53-0.59$ \\
Red & 3 & $0.63-0.69$ & 4 & $0.64-0.67$ \\
Near Infrared - NIR & 4 & $0.76-0.90$ & 5 & $0.85-0.88$ \\
Shortwave Infrared - SWIR1 & 5 & $1.55-1.75$ & 6 & $1.57-1.65$ \\
Shortwave Infrared - SWIR2 & 7 & $2.08-2.35$ & 7 & $2.11-2.29$ \\
\hline
\end{tabular}




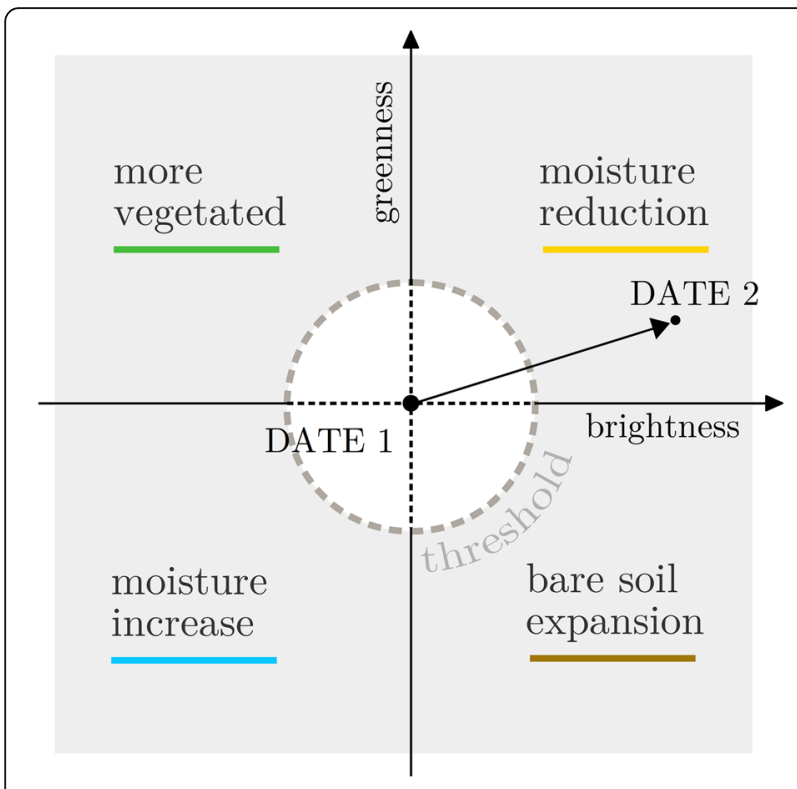

Fig. 2 CVA: meaning of the direction of the change for the four quadrants in the Brightness-Greenness space, considering the vector between two dates. The colors that will be used later on for the classification are also shown

surface characteristics, while the magnitude map gives the amount of the change occurred. The significant change is given by a threshold on the magnitude map, whereas the semantic interpretation is given by the combination of the specific spectral bands or features chosen. In our research we used as a threshold the average of the magnitude values plus the standard deviation, as in other sources [33]. In this way the value has a statistical meaning and the technique can be reproduced by other researchers.

Common choices for the spectral features or bands are in abscissa an indicator of the soil reflectance and in ordinate an indicator of the vegetation vigor. With this choice, the meaning of CVA is provided by four classes given by the four quadrants $\left(0^{\circ}-90^{\circ}, 90^{\circ}-180^{\circ}, 180^{\circ}-\right.$ $270^{\circ}, 270^{\circ}-360^{\circ}$ ) in the bands space, as shown in Fig. 2. In literature, Albedo and NDVI (Normalized Difference Vegetation Index) [34] or the Tasselled Cap features Brightness and Greenness [8] have been used for this purpose. The first quadrant (between $0^{\circ}$ and $90^{\circ}$ ) is indicative of a change towards moisture reduction and in desert areas has been found connected with a change towards salty surfaces, i.e. drying lakes [34]; the fourth quadrant $\left(270^{\circ}-360^{\circ}\right)$, with increasing Brightness and decreasing Greenness, is also connected to a change towards drier conditions, namely towards bare soil/sand expansion and deforestation. The other two quadrants, with decreasing Brightness, are connected with changes towards more wet conditions: the second one $\left(90^{\circ}-180^{\circ}\right)$ with changes towards chlorophyll increase and forest regeneration, and the third $\left(180^{\circ}-270^{\circ}\right)$ with changes towards higher moisture land and water [23, 31]. Notice that CVA gives just a direction of the change, meaning there's no classification of the actual cover types present on the Earth surface. For example, water becoming soil and soil becoming dryer will look the same in the output map (change towards moisture reduction).

TCT coefficients, despite being image independent, are affected by the type of terrestrial surface they've been calculated for, therefore their application to a different type of region might be not appropriate and can affect the results of a multi-temporal analysis [3, 29, 35, 36]. For this work, two sets of desert-adapted TCT for Landsat TOAR data were used. The coefficients are taken from Zanchetta et al. for Landsat 5 [29] and for Landsat 8 [23]. The choice of TOAR data relies on the possibility of comparing the results with TCTs existing in literature for Landsat satellites. Moreover, as pointed out by [27, 37] the use of the TC transform calculated for raw DN data can be not appropriate in change detection studies, being image affected [35], and the use of coefficients calculated for TOAR images is preferable [37].

\section{Multivariate Alteration Detector (MAD) and Maximum Autocorrelation Factor (MAF)}

MAD is a broadly used technique of images linear transformation based on Canonical Correlation Analysis (CCA). It was developed by Nielsen in 1998 [12] in order to improve the simple image differencing technique by "making the images as similar (correlated) as possible, before taking their difference". For this purpose, Nielsen suggests to use standard CCA, as described by Hotelling in 1936 [38]. CCA finds two sets of linear combinations of the original variables, where the first two linear combinations (called canonical variates) are the ones with the largest correlation (called first canonical correlation). The second canonical correlation and canonical variates are determined subject to the condition that they are orthogonal to the first ones, and this process goes on for the higher-order canonical correlations and variates. Performing differences between these pairs of variates allows then to consider a change detection analysis based on linear combinations of the original variables, ordered by correlation (similarity) between pairs [12].

For $\mathrm{N}$ input bands MAD gives $\mathrm{N}$ output difference images, called MAD components. The $\mathrm{MAD}_{\mathrm{i}}$ component corresponds then to $\mathrm{i}$-th difference bearing the maximum variance between two sets of variables that are positively correlated. Each MAD represents a different type of change, supposing that similar types of spatial change will be grouped in one component, since each of them is found under the constraint of being uncorrelated with the preceding one [39]. 
MAF was firstly applied by Nielsen et al. [12] to the MAD components in order to retain the spatial context of the data in the change detection results. In fact MAD components fail to consider the overall spatial coherence of the change, taking into account only local (single pixel) analysis. MAF transform was introduced by Switzer and Green [14] to isolate the noise component of the data, transforming a set of original measurements to a new set of variates where low order variates have maximal spatial autocorrelation, mainly signal, and the highest order variates have minimal spatial autocorrelation, mainly noise.

As such, MAF transformation is a form of Minimum Noise Fraction (MNF) procedure that generates components with maximum Signal to Noise Ratio (SNR): assuming that the noise is derived from the evaluation of the difference between values of neighboring pixels, the first MAF component will exhibit maximum autocorrelation, identified as areas with maximum change, while the noise is expected to be present in lower order components [13].

The first component (MAF1) is the linear combination of the variables that maximizes autocorrelation, the second one (MAF2) is the same type of linear combination with the condition that it is orthogonal to MAF1, and so on, up to the last components, whose number equals the number of initial variables. A complete description of the MAF transformation retrieval is available in [14].

MAF components, like MAD components, are invariant to linear scaling of the input data and can therefore be applied equally on raw data as well as on transformed images. In this research they have been used on TOAR data.

In order to get a value for the significant change, a threshold can be applied to the MAD/MAFs. Since the MADi and MAD/MAF variates are approximately normally distributed about zero and uncorrelated, a good candidate for the thresholding is in terms of standard deviations about the mean for each component. In this sense, all the pixels whose intensities are within $\pm \mathrm{N} \sigma$ of zero are considered unchanged. A usual choice for the threshold is taking values lower and higher than two standard deviations $\pm 2 \sigma$ from the mean $[13,40,41]$. The threshold criterion though is not univocal and more sophisticated criteria based on statistical analysis, like Bayes Rule of Minimum Error [42, 43] and Spectral Mixture Analysis (SMA) [13], have been introduced. For the aim of this study, and missing ground truth data necessary for the SMA, the basic $\pm 2 \sigma$ threshold was chosen.

As pointed out by other authors [7, 40], MAD results contain a high degree of detail, but don't give a univocal possibility for the semantic interpretation.
Therefore, the use of a combined procedure can be a good solution to help in understanding the meaning of the change found by MAD.

\section{Data processing}

The images for both 1984 and 2015 have been processed in Grass GIS using two different regions around the Azraq Oasis and the South Hasakah Lake (see Figs. 3a and 4a for 1984, and 3B and 4B for 2015 images). The raw DN images were firstly transformed to TOAR data with i.landsat.toar, and then to TCT features. The transformation of the TOAR images to TCT features is carried out through Grass GIS BASH scripts encompassing the desert-specific coefficients.

CVA is then performed through a new add-on, i.cva, available for Grass GIS 7.0.x and developed by the author. i.cva gives as output three images, one of the CVA magnitude and two of the CVA angle (one unclassified and one classified by the four quadrants) between two dates. A threshold can also be specified, either by a statistical criterion (number of standard deviation to be summed to the average) or by a customary value. In the case a threshold is set, i.cva gives as output also a map of the final change, given by the classified pixels whose value exceeds the threshold for the magnitude.

For this study, i.cva was run on the Brightness (in abscissa) and Greenness (in ordinate) maps for both dates, taking as a threshold one standard deviation. The outputs of CVA are shown in Figs. 3c, d, e and 4c, d, e separately for the two case studies, where the angle, magnitude and the final change maps are given.

In parallel, MAD/MAF analysis between the 1984 and 2015 images was performed in QGIS on the same areas, using the bands listed in Table 2, to respect the spectral correspondence between the pairs of bands from each satellite. MAD and MAF transformations can be performed in GFOSS using Orfeo Toolbox, available in QGIS environment via the Processing Toolbox. The subsequent processing of the images was conducted in Grass GIS.

\section{Results and discussion}

The research passed through several combinations and tested them on the Oasis case, prior to extensively testing the chosen methodology. Hence an application on the Lake case was carried out.

As a first attempt, a combination of CVA and MAD was considered. The first step was the comparison of several RGB combinations of MAD components in QGIS, in order to select the ones that visually detect the expected change on the surface (Fig. 3f), as commonly performed in MAD application studies. The components 

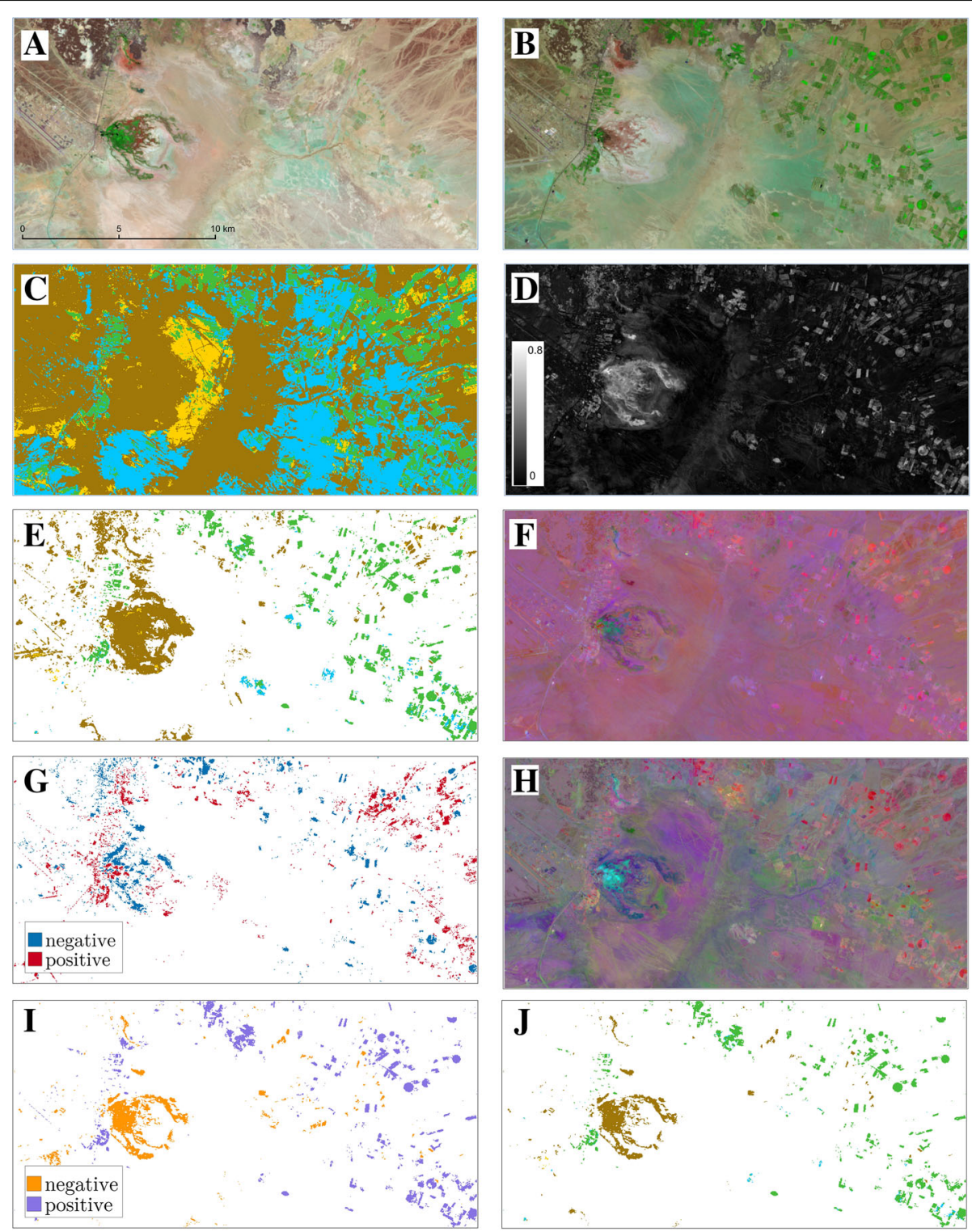

CVA legend: $\square 1^{\text {st }}$ quadrant $\square 2^{\text {nd }}$ quadrant $\square 3^{\text {rd }}$ quadrant $\square 4^{\text {th }}$ quadrant

Fig. 3 Processing of the images for the combined procedure for Al Azraq Oasis. a. RGB composition of Landsat 5 image for 1984; b. RGB composition of Landsat 8 image for 2015; c. CVA classified angle map; $\mathbf{d}$. CVA magnitude map; e. CVA final change map; f. RGB MAD map (components 1,5,6); g. MAD component 5 thresholded and classified map; h. RGB MAF map (variates 1,2,3); i. MAF variate 1 thresholded and reclassified map; $\mathbf{j}$. Final change map. The legend for all the CVA maps is shown below

1, 5 and 6 were selected, and then imported in Grass GIS where the $\pm 2 \sigma$ threshold was applied to each of the three MAD components, giving in output a map of positive and negative values beyond the threshold.

At this point, a visual correspondence was noticed between the CVA results and the thresholded MAD image, where the positive values are generally correlated with the first and forth quadrant (therefore positive Brightness) of the CVA, while the negative values are correlated with the second and third CVA quadrant (therefore negative Brightness). To make this more clear, a new map was created with only two raster categories, grouping all positive values to one and all negative to another (see Fig. $3 g$ for the component 5 as an example). To quantify the similarity between the maps, analogous to visually overlapping the maps, the Grass GIS r.cross module was used, between CVA and two components of MAD. r.cross creates an output raster map representing all unique combinations of category values in the input 

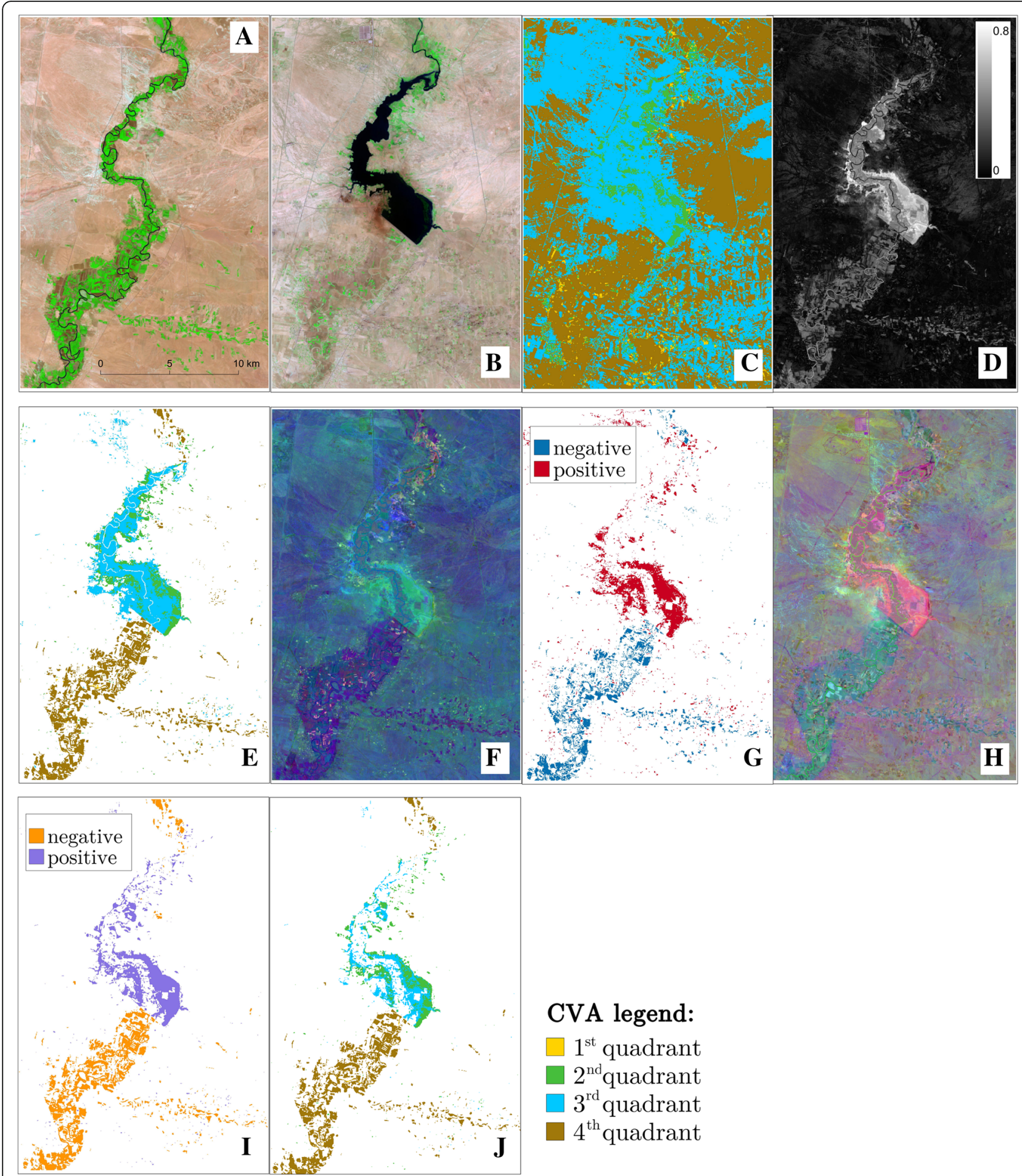

Fig. 4 Processing of the images for the combined procedure for South Hasakah Lake. a. RGB composition of Landsat 5 image for 1984; b. RGB composition of Landsat 8 image for 2015; c. CVA classified angle map; d. CVA magnitude map; e. CVA final change map; f. RGB MAD map (components 1,5,6); g. MAD component 5 thresholded and classified map; h. RGB MAF map (variates 1,2,3); i. MAF variate 1 thresholded and reclassified map; j. Final change map. The legend for all the CVA maps is shown below 
rasters, ${ }^{1}$ and outputs also a table summarizing the occurring categories. The number of possible combinations (not shown here) was still large, and their interpretation was not straightforward.

With reproducibility in mind then, the combination MADCVA is not satisfying, as it depends on the choice of the number and orders of the significant MAD components, that can differ from case to case. Moreover, the high amount of unique combinations between the MAD and CVA classes leaves a complex classification, rather than an easy-to-interpret change map.

Next, the option of the transformation to MAF variates was considered. Like with MAD components, the results contained a high degree of detail, but the meaning of the change was not yet clear (Fig. 3h). The first MAF variate (MAF1), which was imported in Grass GIS and visually compared with the CVA change map, showed a high spatial agreement. It was therefore taken as the only input for the combined methodology. Higher order MAF variates are not shown here but mostly contained noise, as expected.

Like done with MAD, the MAF1 was subsequently thresholded, considering values higher and lower than $\pm 2 \sigma$, and then reduced to a binary valued map grouping positive and negative values (see Fig. 3i). The selection of a single variate as an input alongside the CVA map to r.cross, reduced the number of unique combinations, and now the meaning of the CVA classes could be simply attributed to the MAF1 values. The r.cross categories (in terms of percentage and number of pixels) are shown in Table 3, where the semantic interpretation is retained from the CVA categories (as seen in Fig. 2).

In terms of surface cover, the CVA detects more changed pixels, with an extension up to two or three times more than MAF in some specific classes (see the Bare soil and bare sand expansion class, with more than 5\% pixels undetected from MAF, out of $6.93 \%$ of the CVA). On the other hand, pixels identified by MAF, but not considered from CVA, cover in total only $0.58 \%$ of the Oasis area.

A comparison with the results of a previous CVA change detection analysis, carried out in the same study area and in the same time interval with similar conditions [23], was visually performed. The major difference between the use of the CVA alone and its use combined with MAF is given by the lesser spatial extent of the detected change, as shown by the results in Table 3 . The change classes are correctly detected, but CVA tends to overestimate the change, detecting wider areas, especially in the bare soil expansion class (see Northwestern and Western part of the study area). The combined technique shows more precise identification of the surface elements who changed, in particular the pools and ditches on the East side of the Oasis who went through a drying out process.

Table 3 Results of Grass GIS r.cross module between CVA map and MAF map (first component thresholded and classified for negative and positive values) in terms of pixels count and percentage over the study area for both case studies

\begin{tabular}{|c|c|c|c|c|c|c|}
\hline \multirow[b]{2}{*}{$\begin{array}{l}\text { r.cross } \\
\text { categories }\end{array}$} & \multirow[b]{2}{*}{ CVA classes } & \multirow[b]{2}{*}{$\begin{array}{l}\text { MAF1 } \\
\text { values }\end{array}$} & \multicolumn{2}{|l|}{ Oasis } & \multicolumn{2}{|l|}{ Lake } \\
\hline & & & Pixels count & $\%$ & Pixels count & $\%$ \\
\hline 1 & \multirow[t]{2}{*}{ No data } & Negative & 990 & 0.31 & 1043 & 0.19 \\
\hline 2 & & Positive & 857 & 0.27 & 200 & 0.04 \\
\hline 3 & \multirow[t]{3}{*}{$1^{\text {st }}$ quadrant (Moisture reduction) } & No data & 685 & 0.21 & 143 & 0.03 \\
\hline 4 & & Negative & 39 & 0.01 & 182 & 0.03 \\
\hline 5 & & Positive & $\ldots$ & $\ldots$ & 1 & 0.00 \\
\hline 6 & \multirow[t]{3}{*}{$2^{\text {nd }}$ quadrant (Chlorophyll increase) } & No data & 5551 & 1.73 & 2808 & 0.52 \\
\hline 7 & & Negative & $\ldots$ & $\ldots$ & $\ldots$ & $\ldots$ \\
\hline 8 & & Positive & 8427 & 2.63 & 7406 & 1.37 \\
\hline 9 & \multirow[t]{3}{*}{$3^{\text {rd }}$ quadrant (Higher moisture land and water bodies) } & No data & 1420 & 0.44 & 17215 & 3.19 \\
\hline 10 & & Negative & $\ldots$ & $\ldots$ & $\ldots$ & $\ldots$ \\
\hline 11 & & Positive & 390 & 0.12 & 9882 & 1.83 \\
\hline 12 & \multirow[t]{3}{*}{$4^{\text {th }}$ quadrant (Bare soil and bare sand expansion) } & No data & 16172 & 5.05 & 3645 & 0.68 \\
\hline 13 & & Negative & 6015 & 1.88 & 22480 & 4.16 \\
\hline 14 & & Positive & $\ldots$ & $\ldots$ & $\ldots$ & $\ldots$ \\
\hline \multirow[t]{2}{*}{15} & No data & No data & 279454 & 87.33 & 474995 & 87.96 \\
\hline & & Total & 320000 & 100 & 540000 & 100 \\
\hline
\end{tabular}


The high correspondence of the CVA and MAF results, together with the improvement in the CVA spatial detection, induce to propose the combination of the two techniques as a new methodology for change detection studies, where CVA gives the semantic interpretation and MAF the spatial extent of the change (Fig. 3j).

Considering the new methodology valid, an application to the second case study was implemented, in order to evaluate an antithetical situation, of water replenishment in a semi-arid area. The steps performed in the Oasis case were repeated in the second case, as shown in Fig. $4 \mathrm{c}$-j. Also in this case, the MAD components 1,5 and 6 were chosen for the analysis, but the MAD and CVA combination showed the same weak points seen before. The MAF1 visually exhibited a high spatial correspondence and the Grass GIS r.cross module was applied, giving the results shown in Table 3. The possible combinations were the same seen for the Oasis case, with one stray pixel matching the first CVA quadrant with the positive MAF1. Like in the Oasis case, MAF results are almost entirely overlapped by CVA, with a missed detection of just $0.23 \%$ of the image (compared to the $0.58 \%$ of the Oasis area).

A visual interpretation of the results indicates that the combined methodology finds the abandonment of the fields areas South of the dam along the river banks, and again the MAF reduces the spatial extent of the CVA bare soil expansion class. The expected occurrence of a new vast water basin upstream the dam in the North is also correctly detected. However the MAD/MAF contribution eliminates from the change map the central part of the basin, that was correctly detected by CVA (3.19\% of pixels, belonging to the Higher moisture land and water bodies, not detected by MAF). This issue could be possibly solved if considering higher order MAF variates, and this could be discussed in further work.

Summarizing, the MAD/MAF finds an equivalent change to the CVA and at the same time the CVA allows the physical interpretation of MAD/MAF technique. This gives a new perspective for the use of MAD/MAF, since in other cases found in literature the interpretation of the results is always dependent on the image considered as a case study. The research shows that the use of the two unsupervised techniques in a hybrid approach allows an improvement in understanding the results of both.

For clarification on the performed steps, the proposed workflow for the combined methodology is displayed in Fig. 5.

\section{Conclusions}

A combination of two pixel-based unsupervised change detection techniques for desertification studies was analyzed, taking as case studies two areas in the Middle East region. The first, Al Azraq Oasis, was chosen as a representative of drying conditions, while the second

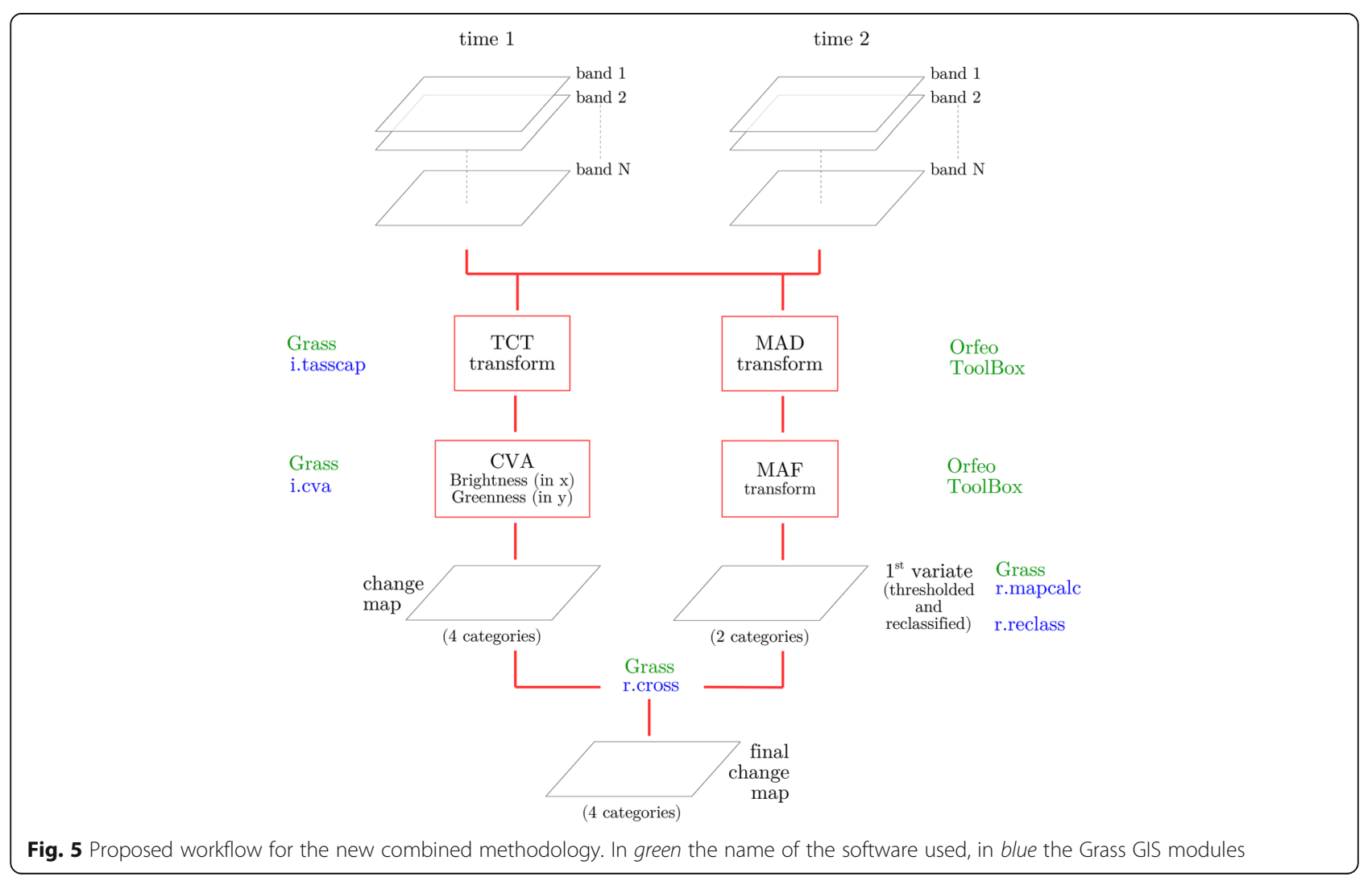


one, South Hasakah Lake, was considered for water bodies replenishment.

A first attempt to combine two change detection techniques was carried out using Change Vector Analysis (CVA) applied to the Tasselled Cap Transform (TCT) features combined with the output of Multivariate Alteration Detector (MAD) analysis. The results, applied to Landsat images between 1984 and 2015, were positive but not satisfying, due to the nature of the MAD components, where the noise is spread among the bands, and to the difficulty in overlapping the many categories given from the two techniques together.

A second approach took into account the Maximum Autocorrelation Factor (MAF) transformation of the MAD components, that groups the images differences, called variates, by decreasing autocorrelation. In this case, one single variate was chosen and combined with the CVA output.

The results of the application to a case previously analyzed by the authors, Al Azraq Oasis, show that the two techniques can be successfully used in a combined way because they give complementary information: while CVA gives a meaning of the surface change, MAF gives a slightly more detailed map of the change on the surface. In this sense, the first MAF variate, appropriately thresholded, can be used like a first step for the identification of the changed features, while a second step is taken assigning a meaning to the features by the CVA semantic classification. This procedure is corroborated by the high correspondence between the change found by the individual techniques: once thresholded and reclassified, MAF positive values match with the first and fourth CVA classes, indicative of changes towards drier conditions, while MAF negative values correspond to the second and third classes of CVA, indicative of changes towards more wet and vegetated conditions.

The results are validated through the comparison with a previous research applying CVA on its own to the same study area surrounding Al Azraq Oasis. The combined methodology detects the expected change on the surface, namely the shrinking of the Oasis and the development of new rural areas, but the MAD/ MAF contributes by more precisely delineating the changed areas. The combined methodology therefore is a conservative procedure, since it excludes a small percentage of pixels from each technique, thus allowing to exclude over-estimation of the change.

Extending the results to the second case study, the expected visual superficial change is again detected by the combined methodology, namely the replenishment of water in the Lake and the loss of cultivated areas along the river. Missing ground truth data, though, an accurate validation of the results can't really be performed in this case. Only the correspondence with the results obtained for Al Azraq Oasis can give some positive feedback about the methodology, beside the fact that the visual change on the surface is correctly identified.

An important output of the research is the improvement in the use of MAD/MAF technique in change detection studies. Previously, the interpretation of the $\mathrm{MAD} / \mathrm{MAF}$ was not straight-forward and in general relied on the single researcher's choices. The research has showed how using a statistical criteria for the threshold determination, and relying on the correspondence between the results of CVA and MAF's first component, the combined technology provides an almost automated, time effective and reproducible technique. As such, it can be used as either a complementary procedure or a first interpretation step in change detection studies. The fast detection can be used for example to perform a preliminary selection of relevant time intervals, on which to perform more accurate change studies with the support of ground data. The methodology then has been found effective for desertification studies in arid and semi-arid areas, detecting the expected change on the surface.

A limitation of the methodology could be given by the choice of the threshold applied to both techniques, that relies on the researcher. Another weak point is that the method has been employed just in a restricted number of cases and could be tested in other geographical regions. Future works can be considered in order to validate the technique, both by verification of the results against ground truth data and by comparing them with other change detection techniques. The implementation of higher order MAF variates could also be taken into account, for a complete investigation of the technique.

An added value of the research is the integration of a CVA calculation module into the Free and Open Source Software (FOSS) project Grass GIS, making the implementation of the technique available to future users.

\section{Endnote \\ ${ }^{1}$ https://grass.osgeo.org/grass72/manuals/r.cross.html}

\section{Authors' contributions}

AZ conceived the idea of the new methodology, carried out the research and wrote the manuscript. GB supervised the process as her guiding PhD professor. Both authors read and approved the final manuscript.

Competing interests

The authors declare that they have no competing interests.

\section{Publisher's Note}

Springer Nature remains neutral with regard to jurisdictional claims in published maps and institutional affiliations. 
Received: 7 December 2016 Accepted: 5 April 2017 Published online: 24 April 2017

\section{References}

1. Singh A. Digital change detection techniques using remotely-sensed data. Int J Remote Sens. 1989;10:989-1003.

2. Coppin P, Lambin EF, Jonckheere I, Muys B. Digital change detection methods in natural ecosystem monitoring: a review. Int J Remote Sens. 2004;25:1565-96.

3. Lu D, Mausel P, Brondizio E, Moran E. Change detection techniques. Int J Remote Sens [Internet]. 2003;25:2365-401. Available from: http://www. informaworld.com/10.1080/0143116031000139863.

4. Blaschke T. Object based image analysis for remote sensing. ISPRS J Photogramm Remote Sens [Internet]. 2010;65:2-16. Available from: http:// dx.doi.org/10.1016/j.isprsjprs.2009.06.004

5. Eastman JR. Change and time series analysis techniques: a review. 1992. p. 1-28.

6. Sui H, Zhou Q, Gong J, Ma G. Processing of multitemporal data and change detection. In: Chen L, Baltsavias Q, editors. Adv. Photogramm. Remote Sens. Spat. Inf. Sci. 2008 ISPRS Congr. B. London: Taylor \& Francis Group; 2008. p. 227-47.

7. Nussbaum S, Menz G. Object-Based Image Analysis and Treaty Verification, New Approaches in Remote Sensing - Applied to Nuclear Facilities in Iran, Edited by S. Nussbaum and G. Menz. Berlin: Springer Science + Business Media B.V; 2008. ISBN: 978-1-4020-6960-4.

8. Malila WA, Lafayette W. Change Vector Analysis : An Approach for Detecting Forest Changes with Landsat. LARS Symp - Purdue University Purdue ePubs [Internet]. 1980;385:326-35. Available from: http://docs.lib.purdue.edu/ lars_symp/385.

9. Flores SE, Yool SR. Sensitivity of change vector analysis to land cover change in an arid ecosystem. Int J Remote Sens [Internet]. 2007;28:1069-88. Available from: http://www.tandfonline.com/doi/abs/10.1080/ 01431160600868482

10. Singh A, Garg PK, Singh S. A Change Vector Analysis Technique for Monitoring of Vegetation Regeneration and Deforestation. Int J Adv Sci Tech Res. 2012;4:329-41.

11. Siwe RN, Koch B. Change vector analysis to categorise land cover change processes using the tasselled cap as biophysical indicator. Environ Monit Assess [Internet]. 2008;145:227-35. Available from: http://www.ncbi.nlm.nih. gov/pubmed/18193332.

12. Nielsen AA, Conradsen K, Simpson JJ. Multivariate Alteration Detection (MAD) and MAF Postprocessing in Multispectral, Bitemporal Image Data : New Approaches to Change Detection Studies. Remote Sens Environ. 1998:64:1-19.

13. Canty MJ. Image Analysis, Classification and Change Detection in Remote Sensing. Boca Raton: CRC Press, Taylor \& Francis Group; 2014. Third Edition. ISBN13: 13: 978-1-4665-7038-2.

14. Switzer P, Green AA. Min/Max Autocorrelation Factors For Multivariate Spatial Imagery. Department of Statistics, Stanford University: National Science Foundation [Internet]. 1984;6. Available at: https://statistics.stanford. edu/sites/default/files/SWI\%20NSF\%2006.pdf.

15. Pannenbecker $A$. Identification of Desertification Indicators Using Bi-Temporal Change detection. In: Braun M, editor. Second Work. EARSeL Spec. Interes. Gr. L. Use L. Cover - Proc. 2006.

16. Nori WTM. Detection of land cover changes in El Rawashda forest, Sudan : A systematic comparison. 2012.

17. Dawelbait MAA, Morari F. LANDSAT, Spetral Mixture Analysis and Change Vector Analysis to Monitor Land Cover Degradation in a Savanna Region in Sudan (1987-1999-2008). Int J Water Resour Arid Environ. 2011;1:366-77.

18. Albalawi EK, Kumar L. Using remote sensing technology to detect, model and map desertification: A review. J Food, Agric Environ. 2013;11:791-7.

19. Shafie $\mathrm{H}$, Seyed MH, Amiri I. Assessment of desertification trends in Sistan Plain, Iran using Rs and GIS. Int J For Soil Eros. 2012;2:97-100.

20. Metternicht Gl, Zinck JA. Remote sensing of soil salinity: Potentials and constraints. Remote Sens Environ. 2003;85:1-20.

21. Huete $A R$, Jackson RD, Post DF. Spectral response of a plant canopy with different soil backgrounds. Remote Sens Environ [Internet]. 1985;17:37-53. Available from: http://www.sciencedirect.com/science/article/pii/0034425785901117.

22. Peel MC, Finlayson BL, McMahon TA. Updated world map of the Koppen-Geiger climate classification. Meteorol Zeitschrift. 2007;15:439-73.

23. Zanchetta A, Bitelli G, Karnieli A. Monitoring desertification by remote sensing using the Tasselled Cap transform for long-term change detection. Nat Hazards. 2016;83:S223-227. Springer Netherlands.
24. Ramsar Convention. Jordan National Report, 7th Meeting of the Conference of the Contracting Parties to the Convention on Wetlands (Ramsar, Iran, 1971). San José: Ramsar Convention; 1999

25. Nguyen H. Agricultural Planning Policy and Variability in Syrian Cereal Production. In: Anderson JR, Hazell PBR, editors. Var. Grain Yields. Baltimore: The Johns Hopkins University Press; 1986. p. 78-90.

26. Hole F. Drivers of unsustainable land use in the Semi-Arid Khabur River Basin. Syria Geogr Res. 2009;47:4-14.

27. Kauth RJ, Thomas GS. The tasselled cap - A graphic description of the spectral-temporal development of agricultural crops as seen by Landsat Proc. Symp. Mach. Process. Remote, Sensed Data, West Lafayette, Indiana, U. S.A, 29 June-1 July 1976. 1976. p. 41-51.

28. Crist EP, Cicone RC. A Physically-Based Transformation of Thematic Mapper Data_-The TM Tasseled Cap. IEEE Trans Geosci Remote Sens. 1984;GE-22:256-63.

29. Zanchetta A, Bitelli G, Karnieli A. Tasselled Cap transform for change detection in the drylands: findings for SPOT and Landsat satellites with FOSS tools. In: Hadjimitsis DG, Themistocleous K, Michaelides S, Papadavid G, editors. Proc. SPIE 9535, Third Int. Conf. Remote Sens. Geoinf. Environ. [Internet]. 2015. Available from: http://proceedings.spiedigitallibrary.org/ proceeding.aspx?doi $=10.1117 / 12.2192597$.

30. Bovolo F, Bruzzone L. A novel theoretical framework for unsupervised change detection based on CVA in polar domain. Int Geosci Remote Sens Symp. 2006;45:379-82.

31. Lorena RB, dos Santos JR, Shimabukuro YE, Brown IF, Kux HJH. A change vector analysis technique to monitor land use/land cover in sw brazilian amazon: acre state. ISPRS Arch [Internet]. 2002;XXXIV:8. Available from: http://www.isprs.org/proceedings/XXXIV/part1/paper/00014.pdf.

32. Bayarjargal Y, Karnieli A, Bayasgalan M, Khudulmur S, Gandush C, Tucker C. A comparative study of NOAA-AVHRR derived drought indices using change vector analysis. Remote Sens Environ [Internet]. 2006;105:9-22. Available from: http://linkinghub.elsevier.com/retrieve/pii/S0034425706002185.

33. He C, Zhao Y, Tian J, Shi P, Huang Q. Improving change vector analysis by cross-correlogram spectral matching for accurate detection of land-cover conversion. Int J Remote Sens. 2013;34:1127-45.

34. Karnieli A, Qin Z, Wu B, Panov N, Yan F. Spatio-Temporal Dynamics of Land-Use and Land-Cover in the Mu Us Sandy Land, China, Using the Change Vector Analysis Technique. Remote Sens [Internet]. 2014;6:931639. Available from: http://www.mdpi.com/2072-4292/6/10/9316/.

35. Ivits E, Lamb A, Langar F, Hemphill S, Koch BS. Orthogonal Transformation of Segmented SPOT IMages: Seasonal and Geographical Dependence of the Tasselled Cap Parameters. Photogramm Eng Remote Sens. 2008;74:1351-64.

36. Yarbrough LD, Easson G, Kuszmaul JS. Proposed workflow for improved Kauth-Thomas transform derivations. Remote Sens Environ [Internet]. 2012;124:810-8. Available from: http://dx.doi.org/10.1016/j.rse.2012.05.003.

37. Huang C, Wylie B, Yang L, Homer C, Zylstra G. Derivation of a tasselled cap transformation based on Landsat 7 at-satellite reflectance. Int J Remote Sens. 2002;23:1741-8.

38. Hotelling H. Relations Between Two Sets of Variates. Biometrika. 1936;28: 321-77. Available from: http://www.jstor.org/stable/2333955.

39. Nori W, Elsiddig EN, Niemeyer I. Detection of land cover changes using multi-temporal satellite. Int Arch Photogramm Remote Sens Spat Inf Sci. 2008;XXXVII:947-52

40. De B-b B, López-caloca AA, Investigación D, Ing G, Tamayo JL. Tropical Dry Forests in the Global Picture: The Challenge of Remote Sensing-Based Change Detection in Tropical Dry Environments. In: Carayannis E, editor. Planet Earth 2011 - Glob. Warm. Challenges Oppor. Policy Pract. InTech. 2011. p. 231-56.

41. Canty MJ, Nielsen AA. Visualization and unsupervised classification of changes in multispectral satellite imagery. Int J Remote Sens. 2006:27:3961-75.

42. Bruzzone L, Prieto DF. Automatic Analysis of the Difference Image for Unsupervised Change Detection. IEEE Geosci Remote Sens. 2000:38:1171-82.

43. Zhang L, Liao M, Yang L, Lin H. Remote Sensing Change Detection Based on Canonical Correlation Analysis and Contextual Bayes Decision. 2007. p. 311-8. 